\title{
Lactobacillus acidophilus attenuates Salmonella-induced intestinal inflammation via TGF- $\beta$ signaling
}

I-Fei Huang ${ }^{1,2,3 \dagger}$, I-Chun Lin ${ }^{4 \dagger}$, Pei-Feng Liu', Ming-Fang Cheng ${ }^{1,2}$, Yen-Chen Liu', Yao-Dung Hsieh ${ }^{6}$, Jih-Jung Chen ${ }^{7}$, Chun-Lin Chen ${ }^{8,9}$, Hsueh-Wei Chang ${ }^{10,11^{*}}$ and Chih-Wen Shu ${ }^{5^{*}}$

\begin{abstract}
Background: Salmonella is a common intestinal pathogen that causes acute and chronic inflammatory response. Probiotics reduce inflammatory cytokine production and serve as beneficial commensal microorganisms in the human gastrointestinal tract. TGF- $\beta$ (transforming growth factor $\beta$ )/SMAD and NF-KB signaling play important roles in inflammation in intestinal cells. However, the involvement of the signaling in regulating inflammation between Salmonella and probiotics is not fully understood.
\end{abstract}

Methods: L. acidophilus and prebiotic inulin were used to treat human intestinal Caco-2 cells prior to infection with Salmonella. The cells were harvested to examine the cytokines and MIR21 expression with immunoblotting and real-time PCR. NF-KB and SMAD3/4 reporter vectors were transfected into cells to monitor inflammation and TGF- $\beta 1$ signaling, respectively.

Results: In this study, we showed that the probiotic L. acidophilus decreased Salmonella-induced NF-KB activation in human intestinal Caco-2 cells. Expression of the inflammatory cytokines, TNF-a and IL-8, in L. acidophiluspretreated cells was also significantly lower than that in cells infected with Salmonella alone. Moreover, TGF- $\beta 1$ and MIR21 expression was elevated in cells pretreated with L. acidophilus or synbiotic, a combination of inulin and $L$. acidophilus, compared to that in untreated cells or cells infected with S. typhimurium alone. By contrast, expression of SMAD7, a target of MIR21, was accordingly reduced in cells treated with L. acidophilus or synbiotics. Consistent with TGF- 31 /MIR21 and SMAD7 expression, SMAD3/4 transcriptional activity was significantly higher in the cells treated with L. acidophilus or synbiotics. Furthermore, TGF- $\beta 1$ antibody antagonized the SMAD3/4 and NF-KB transcriptional activity modulated by L. acidophilus in intestinal cells.

Conclusion: Our results suggest that the TGF- $31 /$ MIR21 signaling pathway may be involved in the suppressive effects of $L$. acidophilus on inflammation caused by S. typhimurium in intestinal Caco-2 cells.

Keywords: L. acidophilus, Synbiotics, Salmonella, TGF-b, NF-KB, MIR21, SMAD

\section{Background}

The human intestine houses a dense and diverse microbial community, which is estimated to contain at least 500 different species of bacteria. These microbes, collectively referred to as the commensal microbiota, play

\footnotetext{
* Correspondence: changhw2007@gmail.com; cwshu@vghks.gov.tw

${ }^{\dagger}$ Equal contributors

${ }^{10}$ Department of Biomedical Science and Environmental Biology, Kaohsiung

Medical University, Kaohsiung, Taiwan

${ }^{5}$ Department of Medical Education and Research, Kaohsiung Veterans

General Hospital, Kaohsiung, Taiwan

Full list of author information is available at the end of the article
}

important roles in human health, such as processing of nutrients, regulation of fat storage [1,2], and protection against pathogens [3]. Salmonella typhimurium (S. typhimurium), an intestinal pathogenic bacterium, is one of the most common non-typhoidal Salmonella (NTS), which is the leading cause of acute food-borne disease [4]. Salmonellosis results in diarrhea, vomiting, and fever in the majority of people after infection. Approximately 400 persons die each year because of severe Salmonellosis, particular young children, the elderly, and the immunocompromised persons. S. typhimurium penetrates 
intestinal cells and macrophages to induce intestinal inflammation using two type III secretion systems (T3SSs) [5]. S. typhimurium induces NF-kB activation and the secretion of pro-inflammatory cytokines, such as interleuckin-8 (IL-8) [6] and tumor necrosis factor alpha (TNF- $\alpha$ ) [7]. The excessive inflammatory response may help $S$. typhimurium eliminate other competitive microbiota in the host [8].

Probiotics attenuate NF- $\mathrm{kB}$ activation and inflammatory cytokine production in intestinal epithelia cells $[9,10]$ and in vivo [11-13]. These probiotics are considered beneficial commensal microorganisms in human gastrointestinal tract $[14,15]$. TGF- $\beta$ was identified as a negative regulator of NF- $\mathrm{kB}$ activation in gut inflammation [16]. TGF- $\beta$ activates SMAD2/3 and initiates SMAD4 dependent transcription to induce $\mathrm{I} \kappa \mathrm{B} \alpha$, inhibitor of NF- $\mathrm{B}$, and attenuate inflammatory responses [16], whereas SMAD7 physically inhibits the TGF- $\beta$ receptor and induces the pro-inflammatory pathways mediated by NF-kB [17]. Our previous study showed that probiotics enhance enteric protection against pathogens and reduce mucosal inflammation by enhancing tumor growth factor beta (TGF- $\beta$ ) signaling in vivo [18]. Recent reports also demonstrate that TGF- $\beta$ induces microRNA-21 (MIR21) expression, which targets 3'-UTR of SMAD7 to attenuate its expression and enhances TGF- $\beta$ signaling [19-21]. Furthermore, probiotics decrease SMAD7 expression and elevate IкB $\alpha$ expression to suppress pathogen-induced inflammation in human gastric epithelia cells [9], suggesting that feed-back control between TGF- $\beta$ and SMAD7 may be crucial for inflammation.

Although several studies demonstrated the suppression of inflammation using probiotics, the TGF- $\beta$ mediated signaling caused by probiotics administration on $S$. typhimurium-induced inflammation in human intestinal cells remains unclear. In this study, we examined the role of TGF- $\beta$ signaling molecules in human intestinal Caco-2 cells infected with $S$. typhimurium in the absence or presence of the probiotic $L$. acidophilus alone or combination with the prebiotic inulin, a non-digestible oligosaccharide that enhances the growth of probiotics. We found that TGF- $\beta$ and MIR21 expression is induced, whereas SMAD7 expression was decreased in human colorectal Caco-2 cells pretreated with $L$. acidophilus compared to cells infected with $S$. typhimurium alone. SMAD3/4 transcriptional activity was further elevated in L. acidophilus-treated cells, consistent with reduced NF-kB transcriptional activity and inflammatory cytokines induction, including IL- 8 and TNF- $\alpha$. Therefore, our findings elucidate the effect of TGF- $\beta$ signaling on probiotics or synbiotics, which may identify a new treatment strategy for inflammation caused by Salmonella infection using probiotics.

\section{Methods}

\section{Cell culture}

The human colonic adenocarcinoma cell line (Caco-2, BCRC 60182) was used in this study and cultured in Dulbecco's modified Eagle's medium (DMEM) (Invitrogen, 12100-046) supplemented with 3.7 g/L NaHCO3 (Merck), $1.7 \mathrm{mM}$ glutamine (Brunschwig Chemie BV), $100 \mathrm{U} / \mathrm{ml}$ penicillin, $100 \mu \mathrm{g} / \mathrm{ml}$ streptomycin, $0.25 \mu \mathrm{g} /$ $\mathrm{ml}$ amphotericin (Biological Industries), $0.01 \mathrm{mg} / \mathrm{ml} \mathrm{hu-}$ man transferrin (T-0665, Sigma-Aldrich), $1.0 \mathrm{mM}$ sodium pyruvate, and $10 \%(\mathrm{v} / \mathrm{v})$ fetal bovine serum (Biological Industries) at $37{ }^{\circ} \mathrm{C}$ in a humidified atmosphere of $5 \%(\mathrm{v} / \mathrm{v}) \mathrm{CO}_{2}$ in air. Cells were seeded in $75 \mathrm{~cm}^{2}$ tissue culture flasks (Corning Incorporated) containing $8 \mathrm{ml}$ DMEM medium. This study encompassed 10 cell passages of the cell line ranging from the 20th to the $29^{\text {th }}$ passage.

\section{S. typhimurium culture and infection}

The enteric pathogen S. typhimurium (BCRC 10747) was grown on a blood agar plate (BAP, trypticase soy agar with $5 \%$ sheep blood) (CMP Ltd.) at $37{ }^{\circ} \mathrm{C}$ overnight. The bacterial colonies were collected with disposable L-shaped cell spreaders in PBS (Biological Industries). The collected bacteria were washed, centrifuged at 1,5000 $\times \mathrm{g}$ for $15 \mathrm{~min}$ at $4{ }^{\circ} \mathrm{C}$ and resuspended in PBS. The concentration of $S$. typhimurium was measured using a spectrophotometer as the optical density (OD) at $600 \mathrm{~nm}$. For bacterial infection, Caco- 2 cells $\left(1 \times 10^{6}\right.$ cells/well $)$ were incubated with various concentrations $S$. typhimurium, ranging from $1 \times 10^{4} \mathrm{CFU} / \mathrm{ml}$ to $1 \times 10^{10} \mathrm{CFU} / \mathrm{ml}$ in antibiotic-free DMEM for $1 \mathrm{~h}$ at $37{ }^{\circ} \mathrm{C}$. The cells were washed with PBS and recovered with DMEM media containing gentamicin $(50 \mu \mathrm{g} / \mathrm{ml}$, Sigma-Aldrich) for the following experiments.

\section{Treatment of probiotic L. acidophilus}

The probiotic $L$. acidophilus (BCRC 10695) was cultured in DeMan, Rogosa, Sharpe (MRS) broth (CMP Ltd.) at $37{ }^{\circ} \mathrm{C}$ overnight. After centrifugation at $15000 \times \mathrm{g}$ for $15 \mathrm{~min}$ at $4{ }^{\circ} \mathrm{C}$, the collected bacteria were washed once with PBS (Biological Industries, 02-023-1) and subsequently resuspended with PBS for the treatment of Caco- 2 cells. Caco- 2 cells $\left(1 \times 10^{6}\right.$ cells/well $)$ were seeded in a 6-well plate and treated with L. acidophilus $(2 \times$ $10^{7} \mathrm{CFU} / \mathrm{ml} /$ well, $\mathrm{MOI}=20$ ) in the presence or absence of prebiotic ( $1 \%$ inulin, Orafti $\left.{ }^{\circ} \mathrm{GR}\right)$ or probiotic in antibiotic-free DMEM for $1 \mathrm{~h}$. Subsequently, the Caco-2 cells were infected with $S$. typhimurium $\left(1 \times 10^{7} \mathrm{CFU}\right)$ for $1 \mathrm{~h}$ and recovered with DMEM media containing gentamicin $(50 \mu \mathrm{g} / \mathrm{ml}$, Sigma-Aldrich) for the following experiments. 
Transfection and luciferase reporter assay

To test the SMAD and NF- $\mathrm{kB}$ transcriptional activity, the Caco-2 cells in a 384-well plate were transfected with $50 \mathrm{ng}$ of plasmid containing NF-kB-responsive reporter vector pGL4.32 (Promega) or SMAD responsive reporter vector pGL4.48 (Promega), which contains the NF- $\mathrm{kB}$ and SMAD-binding sites, respectively. The pGL4.50 (Promega) constitutively expressing luciferase was used as a normalization control. At $20 \mathrm{~h}$ post transfection, the cells were infected with S. typhimurium in the absence of presence of probiotic or synbiotic for $1 \mathrm{~h}$. The cells were washed once with PBS and recovered with DMEM media containing gentamicin ( $50 \mu \mathrm{g} / \mathrm{ml}$, Sigma-Aldrich). The luminescence of the luciferase-based reporter was measured with DMEM medium containing $200 \mu \mathrm{M}$ D-luciferin (Promega). The results were read using a Fluoroskan Ascent FL reader (Thermo Fisher Scientific).

\section{Protein extraction and western blot}

Human colorectal epithelia Caco-2 cells were briefly rinsed in PBS (Biological Industries) and lysed with RIPA buffer (1 \% NP40 [MDBio, 101-9016-45-9], 50 mM Tris $\mathrm{HCl}, \mathrm{pH} 7.5,150 \mathrm{mM} \mathrm{NaCl}, 0.25 \%$ sodium deoxycholate [Sigma-Aldrich, D6750], $0.1 \%$ sodium dodecyl sulfate [SDS; Calbiochem, 428015], and protease inhibitor cocktail [Roche, 11873580001]). The cell lysates were centrifuged at $13200 \times \mathrm{g}$ for $30 \mathrm{~min}$ and the supernatant was collected to determine the protein concentration using Bradford Reagent (Sigma-Aldrich). The cell lysates were resolved by SDS-PAGE and transferred onto nitrocellulose membranes using a wet electrophoretic transfer system (Bio-Rad). The membrane was blocked with $5 \%$ skim milk and then incubated with primary antibodies against SMAD7 (sc-11392) (Santa Cruz Biotech), TGF- $\beta$ (abcam) and actin (Sigma-Aldrich) to determine the protein expression using the ChemiDoc XRS Imaging System (Bio-Rad) and analysis with Image Lab4.1 (Bio-Rad).

\section{Real-Time PCR}

The cells transfected with siRNA were used to extract the total RNA with TRIzol Reagent (Invitrogen, 15596-018). A total of $1 \mu \mathrm{g}$ RNA was reverse-transcribed with SuperScript II RNase H-Reverse Transcriptase (Invitrogen, 18064-014) for cDNA synthesis. The amounts of IL-8, TNF- $\alpha$ and MIR21 mRNA relative to S-26 were analyzed by real-time PCR performed in a StepOnePlus s'" system (Applied Biosystems) with the SYBR Green Master Mix (Applied Biosystems, 4385612). The primers for the genes are as follows: IL-8 forward 5' - ACTGAGAGTGATTGAGAGT GGAC-3' and reverse 5' - AACCCTCTGCACCCAGTTT TC -3 ', TNF- $\alpha$ forward 5 ' - GAGGCCAAGCCCTGGG ATG $-3^{\prime}$ and reverse $5^{\prime}$ - CGGGCCGATTGATCTCA GC -3', S-26 forward 5' - CCGTGCCTCCAAGATGA CAAAG $-3^{\prime}$ and reverse $5^{\prime}$ - GTTCGGTCCTTGCG
GGCTTCAC - 3', and MIR21 reverse transcription 5' - CTCAACTGGTGTCGTGGAGTCGGCAATTCAG TTGAGTCAACATC $-3^{\prime}$ and gene specific forward 5' - CGGCGGTAGCTTATCAGACTGA -3'.

\section{Data analysis}

Data were statistically analyzed by Prism 5.0 (GraphPad) using one-way ANOVA followed by Tukey's multiple comparison test to compare the effects between each group. All results are expressed as the mean \pm SEM from at least 3 individual experiments. The lev el of significance was set at $P<0.05$ ( $P$-value $\leqq 0.05$ considered significant (*), $P$-value $\leqq 0.01$ considered highly significant $(* *)$, and $P$-value $\leqq 0.001$ considered extremely significant $\left.\left({ }^{* * * *}\right)\right)$.

\section{Results}

L. acidophilus attenuates S. typhimurium-induced inflammatory response in Caco- 2 cells

NF- $\mathrm{kB}$ transcribes global genes involved in inflammation, such as TNF- $\alpha$ [22] and IL-8 [23]. To explore the effect of probiotic on S. typhimurium-induced inflammation, an NF- $\mathrm{KB}$ responsive reporter assay was used to optimize the infected concentration of S. typhimurium for human intestinal cells. Human intestinal Caco-2 cells were transfected with NF- $\mathrm{kB}$ responsive reporter vector for $16 \mathrm{~h}$ and then incubated with S. typhimurium, ranging from $1 \times 10^{6} \sim 1 \times 10^{10} \mathrm{CFU} / \mathrm{ml}$ (MOI 1 to 10000), in antibiotic-free media for $1 \mathrm{~h}$ (Fig. 1). The infected Caco-2 cells were recovered with media containing gentamicin to monitor NF- $\mathrm{KB}$ transcriptional activity at $6 \mathrm{~h}$ or $24 \mathrm{~h}$ post-infection. NF- $\mathrm{kB}$ transcriptional activity was significantly increased 2.5- and 3-fold in Caco-2 cells infected with S. typhimurium at $6 \mathrm{~h}$ post-infection in a concentration of $1 \times 10^{6} \mathrm{CFU} / \mathrm{ml}(p \leqq 0.05)$ and $1 \times$ $10^{7} \mathrm{CFU} / \mathrm{ml}(p \leqq 0.01)$, respectively (Fig. 1a). Therefore, the optimal ratio for S. typhimurium to human colorectal epithelia Caco-2 cells is an MOI 10 for this study. Moreover, S. typhimurium entered host cells at $3 \mathrm{~h}$ after infection (Additional file 1: Figure S1), suggesting that $S$. typhimurium triggered inflammatory response may be mainly resulted from extracellular stimuli, most likely are the lipopolysaccharides of S. typhimurium. Because L. acidophilus is one of the most commonly used probiotics in the food supply, we used $L$. acidophilus as a probiotic for the following experiments. Further, inulin is a well-known prebiotic that is used to improve the growth and viability of probiotics in fermented milk [24]. Therefore, we employed human intestinal Caco-2 cells harboring NF- $\mathrm{kB}$ responsive reporter vector to infect with $S$. typhimurium (MOI 10) without or with inulin or L. acidophilus (MOI 20) or combination (synbiotic). Both L. acidophilus (MOI 20) and synbiotic significantly suppressed 

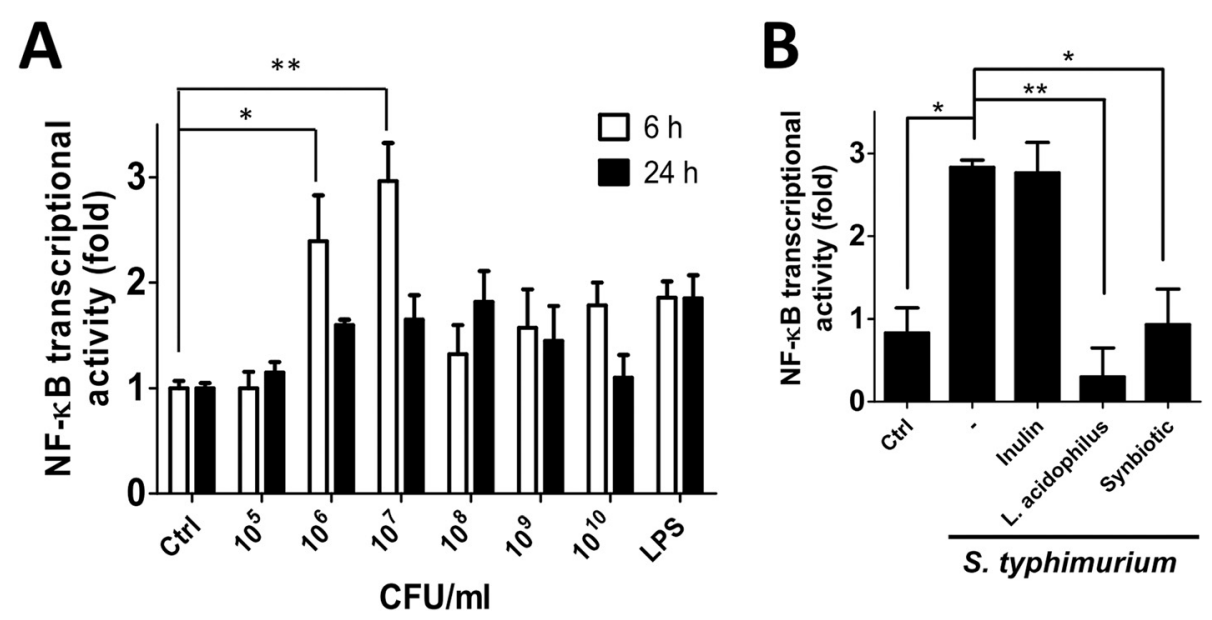

Fig. 1 Effects of L. acidophilus on S. typhimurium-induced NF-KB activation. a Human intestinal Caco-2 cells were transfected with a luciferase reporter vector for NF-KB or CMV (normalization control) overnight and then infected with various concentrations of S. typhimurium as indicated, ranging from $10^{4}$ to $10^{10} \mathrm{CFU} / \mathrm{ml}$ in antibiotic-free DMEM for $1 \mathrm{~h}$ at $37^{\circ} \mathrm{C}$. The cells were recovered with DMEM media containing gentamicin $(50 \mathrm{\mu g} / \mathrm{ml})$ and NF-KB transcriptional activity was measured at 6 or $24 \mathrm{~h}$ post-infection. $\mathbf{b}$ For treatment with L. acidophilus, transfected cells were treated with $1 \%$ inulin or L. acidophilus (L. acidophilus:S. typhimurium =2:1) or synbiotics (L. acidophilus and $1 \%$ inulin) $1 \mathrm{~h}$ prior to infection of S. typhimurium as mentioned above. The cells were added with DMEM medium containing D-luciferin and antibiotic at $6 \mathrm{~h}$ post-infection to measure NF-KB activity. The data were analyzed with Prism 5, and the results are shown as the means \pm SEM from three independent experiments

NF-kB transcriptional activity in cells infected with $S$. typhimurium ( $p \leqq 0.05$ ) (Fig. 1b), whereas inulin alone did not alter S. typhimurium-induced NF-kB transcriptional activity. Furthermore, the mRNA level of IL-8 and TNF- $\alpha$, inflammatory cytokines and transcripts of NF$\mathrm{KB}$, were significantly reduced in $L$. acidophilus and synbiotic pretreated Caco-2 cells compared to the cells infected with $S$. typhimurium alone (Fig. 2) ( $p \leqq 0.05)$, suggesting that the L. acidophilus and synbiotic decrease NF-kB activation, which in turn inhibits inflammatory cytokine induction and reduces the inflammation in human intestinal cells during $S$. typhimurium infection.

\section{L. acidophilus induces TGF- $\beta$ and MIR21 expression}

TGF- $\beta$, an important suppressor of inflammation, is induced by $L$. acidophilus treatment in a mouse model [18], whereas TGF- $\beta$ expression did not change in human gastric epithelial cells [9]. The role of TGF- $\beta$ in $L$. acidophilus-treated human colorectal epithelia Caco-2

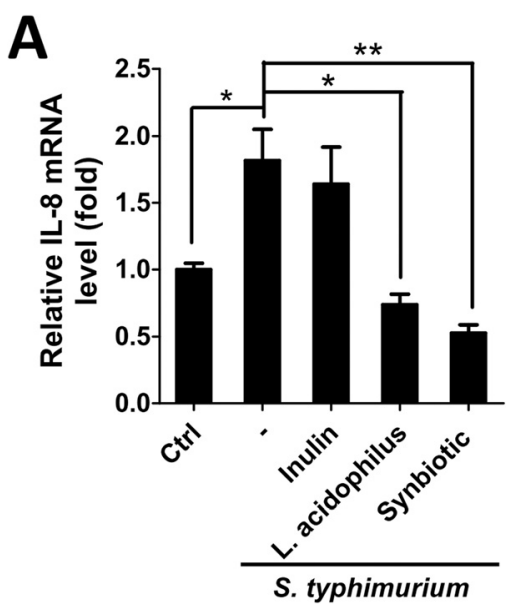

B

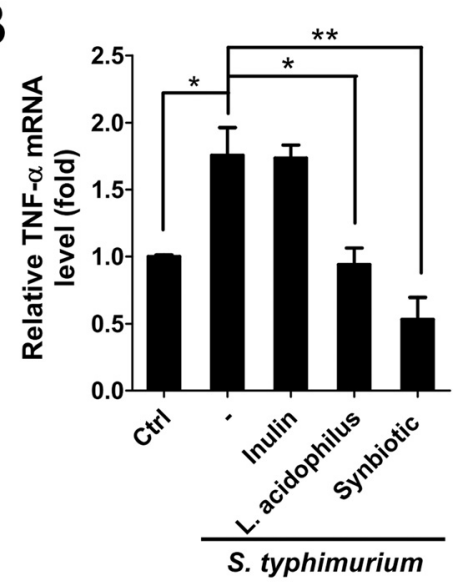

Fig. 2 Effects of L. acidophilus and S. typhimurium on IL-8 and TNF-a mRNA expression. Human intestinal Caco-2 cells were treated with $1 \%$ inulin or L. acidophilus (L. acidophilus:S. typhimurium $=2: 1$ ) or synbiotics (L. acidophilus and $1 \%$ inulin) $1 \mathrm{~h}$ prior to infection with S. typhimurium in DMEM without antibiotic for $1 \mathrm{~h}$. The cells were harvested at $6 \mathrm{~h}$ post-infection for mRNA isolation. The isolated mRNA was further used to determine the expression of (a) IL-8 and (b) TNF-a by quantitative PCR (qPCR). The data are shown as the means \pm SEM from three independent experiments 
cells is not fully understood. To determine whether TGF- $\beta$ is involved in the anti-inflammation of $L$. acidophilus in Caco-2 cells during S. typhimurium infection, TGF- $\beta$ expression in treated cells was assessed by immunoblotting (Fig. 3a). Both L. acidophilus and synbiotic increased TGF- $\beta$ expression in human intestinal Caco-2 cells during $S$. typhimurium infection. Moreover, TGF- $\beta$ enhances MIR21 maturation in primary cultured fibroblasts [19]; therefore, we further evaluated MIR21 expression in the treated Caco-2 cells (Fig. 3b). Consistently, MIR21 expression was increased in cells with L. acidophilus or synbiotic, but not in the other treated cells, suggesting that the TGF- $\beta$ /MIR21 axis may be involved in the anti-inflammatory effects of $L$. acidophilus in Caco-2 cells post-infected with S. typhimurium.

\section{L. acidophilus increases TGF- $\beta$ /SMAD signaling in intes- tinal cells}

MIR21 targets 3'-UTR of SMAD7 and attenuates gene expression [19], which may augment TGF- $\beta$ signaling for SMAD3/4 transcriptional activity, thereby playing an important role in this positive feedback loop to suppress inflammation. The protein expression of SMAD7 in $S$. typhimurium infected cells with or without $L$. acidophilus was determined by immunoblotting (Fig. 4a). The expression of SMAD7 was significantly increased in cells infected with $S$. typhimurium compared to the uninfected cells, whereas the cells pretreated with $L$. acidophilus or synbiotic had a reduction in the increased expression of SMAD7 (Fig. 4a). We further evaluated the SMAD3/ 4 transcriptional activity with the cells harboring SMAD3/4 responsive reporter vector. L. acidophilus induced SMAD3/4 activity in intestinal cells with either live or UV-inactivated S. typhimurium or LPS (Additional file 2: Figure S2). Moreover, SMAD3/4 transcriptional activity was increased 7 -fold and 2.5 -fold in cells administered with $L$. acidophilus and synbiotics, respectively (Fig. 4b). Pretreatment with synbiotics resulted in significantly less activation of SMAD3/4 compared to the cells treated with $L$. acidophilus alone, suggesting that the combination of inulin and $L$. acidophilus has additional effects on TGF- $\beta$ /SMAD signaling. In addition, the cells pretreated with TGF- $\beta$ antibody modestly diminished the effects of L. acidophilus on SMAD3/4 and NF- $\mathrm{KB}$ transcriptional activity (Fig. 5), supporting the hypothesis that $L$. acidophilus activates TGF- $\beta$ signaling to suppress inflammation caused by $S$. typhimurium in intestinal cells.

\section{Discussion}

Human immunity plays an important role in the development of more serious clinical diseases after S. typhimurium infection because of increased pro-inflammatory cytokine expression in the intestine [25]. S. typhimurium infection activates NF- $\mathrm{KB}$ in intestinal epithelium cells and subsequently up-regulates gene transcription of proinflammatory cytokines, such as IL-8 [26] and TNF- $\alpha$ [22]. The present study demonstrates that S. typhimurium

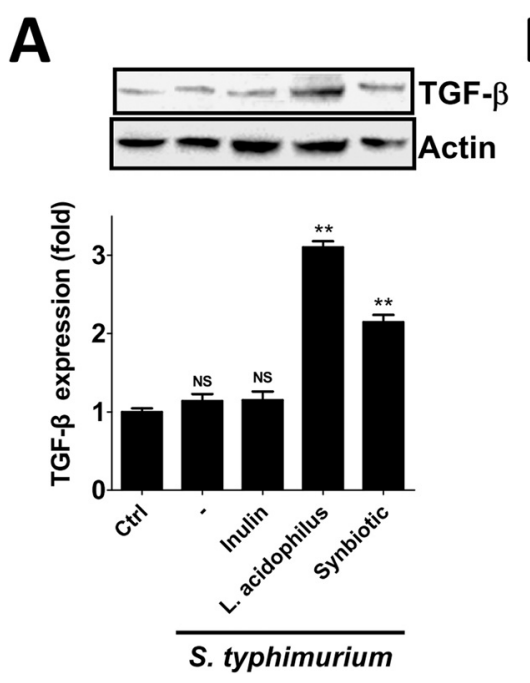

B

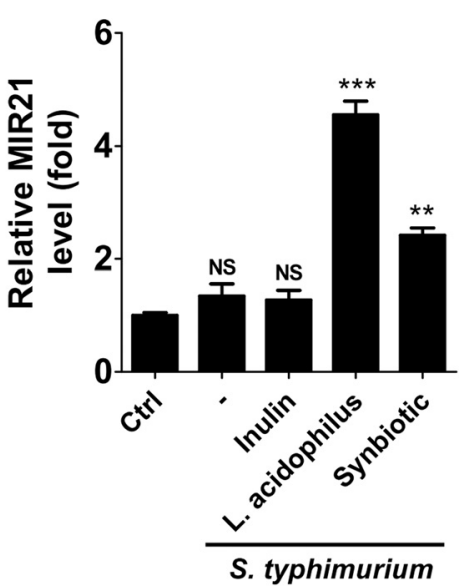

Fig. 3 Effects of L. acidophilus and S. typhimurium on TGF- $\beta$ and MIR21 expression. Human intestinal Caco-2 cells were treated with $1 \%$ inulin or L. acidophilus (L. acidophilus : S. typhimurium $=2: 1$ ) or synbiotics $1 \mathrm{~h}$ prior to infection with S. typhimurium in DMEM without antibiotic for 1 h. The cells were harvested at $6 \mathrm{~h}$ post-infection for protein extraction. a The cells were lysed to extract the proteins and assess TGF- $\beta$ expression by immunoblotting. $\mathbf{b}$ The treated cells were harvested at $6 \mathrm{~h}$ post-infection for total RNA isolation. The isolated RNA was further used to determine the expression of MIR21 by quantitative PCR (qPCR). The results are from three independent experiments, and the data are shown as the means \pm SEM. NS: not significant 


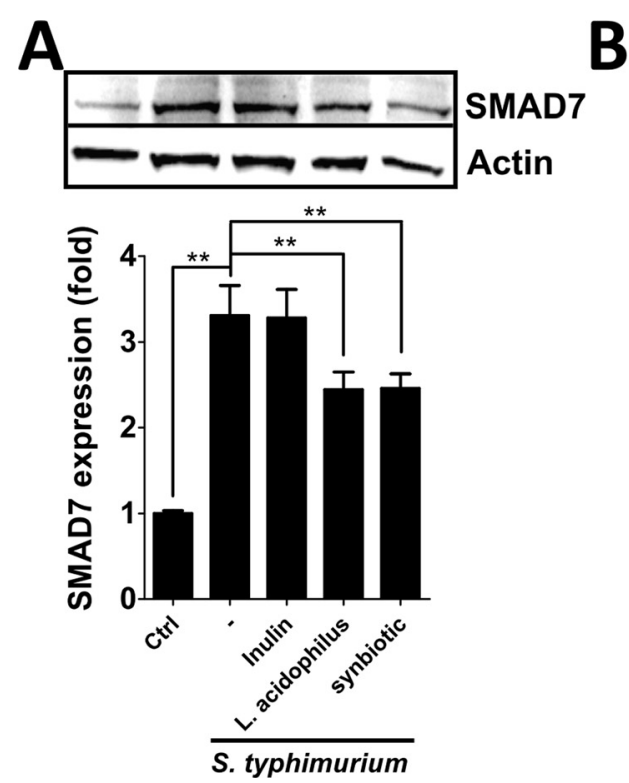

B

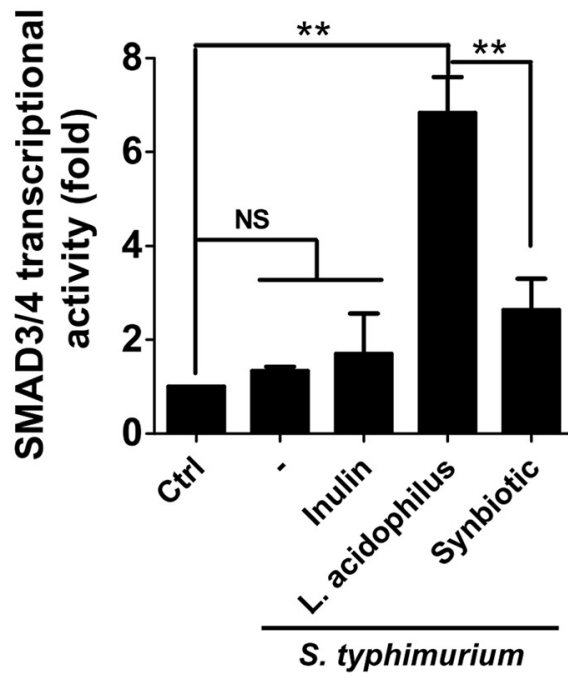

Fig. 4 Effects of L. acidophilus and S. typhimurium on SMAD7 expression and SMAD3/4 transcriptional activity. a Human intestinal Caco-2 cells were treated with $1 \%$ inulin or L. acidophilus (L. acidophilus:S. typhimurium =2:1) or synbiotics (L. acidophilus and $1 \%$ inulin) 1 h prior to treatment with S. typhimurium in DMEM without antibiotic for $1 \mathrm{~h}$. The cells were harvested at $6 \mathrm{~h}$ post-infection for protein extraction. SMAD7 protein expression was determined by immunoblotting. $\mathbf{b}$ Human intestinal Caco-2 cells were transfected with luciferase reporter plasmid for SMAD3/4 or CMV (normalization control) overnight and then treated or infected as above. The cells were added DMEM medium containing D-luciferin at $6 \mathrm{~h}$ post-infection to read the signal with a Luminometer. The quantitative results are shown as the means \pm SEM from three independent experiments. NS: not significant

infection induces TNF- $\alpha$ and IL-8 pro-inflammatory cytokine expression in human intestinal Caco-2 cells. The previous study demonstrated that L. acidophilus pretreatment decreases S. typhimurium induced inflammation [7]. The finding is consistent with the results showing that anti-inflammatory effects are achieved by probiotics in clinical setting [27]. In normal intestinal mucosal cells, the TGF- $\beta$ signal may negatively regulate NF$\kappa B$ activation by stimulating the negative regulator, I $\mathrm{KB} \alpha$ [16]. Silencing SMAD7 can restore TGF $\beta /$ SMAD3 signaling and result in the suppression of inflammatory cytokine production in patients with inflammatory bowel
A

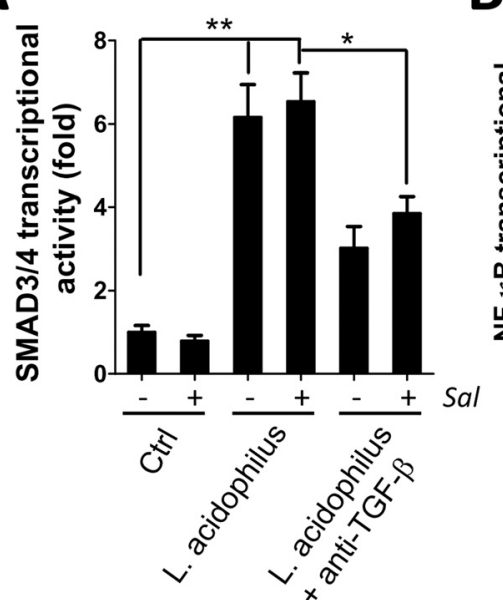

B

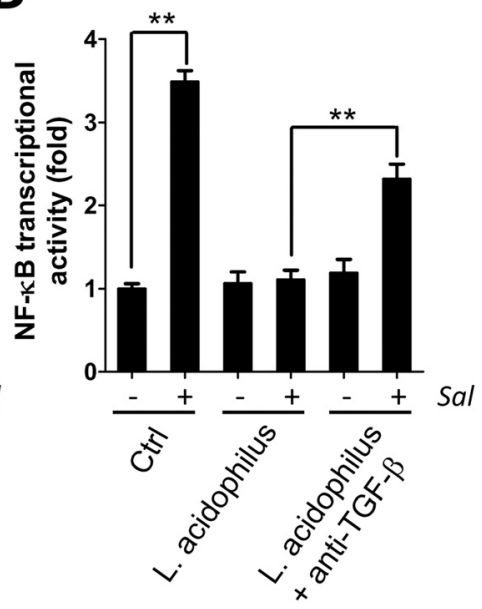

Fig. 5 Effects of TGF- $\beta$ on L. acidophilus regulated SMAD3/4 and NF-kB transcriptional activity. Human intestinal Caco-2 cells were transfected with luciferase reporter plasmid for a SMAD3/4 or $\mathbf{b}$ NF-KB overnight and then treated with L. acidophilus (L. acidophilus:S. typhimurium = 2:1) or L. acidophilus mixed with anti-TGF- $\beta$ antibody $(1 \mu \mathrm{g} / \mathrm{ml}) 1 \mathrm{~h}$ prior to infection with S. typhimurium. The cells were recovered and added DMEM medium containing D-luciferin at $6 \mathrm{~h}$ post-infection to read the signal with a Luminometer. The results are normalized with the cells harboring luciferase constitutively expressed vector (CMV) and are shown as the means \pm SEM from three independent experiments 
diseases [28]. Our results reveal that L. acidophilus induces TGF- $\beta$ /MIR21 expression and down-regulates SMAD7 expression, which may decrease NF-kB-activated inflammation caused by $S$. typhimurium in human intestinal Caco- 2 cells.

A prebiotic was first defined as a non-digestible food ingredient that beneficially affects the host by selectively stimulating the growth and/or activity of one or a limited number of bacteria in the colon and improving host health [29]. Preliminary research on prebiotics demonstrated potential effects on calcium and other mineral absorption [30], immune system effectiveness [31], bowel $\mathrm{pH}$, reduction of colorectal cancer risk [32], and inflammatory bowel disorders, such as Crohn's disease and ulcerative colitis [33]. Additionally, Roberfroid stated that only two particular prebiotics then fully meet this definition, including trans-galactooligosaccharide and inulin [29]. Inulin, a long-chain prebiotic, stimulates the growth of probiotics [29]. Our results showed that combination of probiotic and inulin as synbiotic had a suppressive effect on the $S$. typhimurium-induced inflammatory cytokine production in human intestinal Caco-2 cells, including IL-8 and TNF- $\alpha$. However, synbiotics showed a smaller effect on TGF- $\beta$ and MIR21 expression, and SMAD3/4 transcriptional activity compared to the cells treated with $L$. acidophilus alone during infection with S. typhimurium. Previous report indicates that prebiotic oligosaccharides reduce proinflammatory cytokines in intestinal Caco-2 cells via activation of PPARy and peptidoglycan recognition protein 3 [34]. These results suggest that inulin may trigger additional signaling events to prevent $S$. typhimurium-induced inflammation in a TGF- $\beta$ independent manner.

S. typhimurium is an intracellular bacterium that enters cells after $2 \mathrm{~h}$ of infection. We infected Caco-2 cells with $S$. typhimurium for only $1 \mathrm{~h}$ and recovered the cells with media containing gentamicin. Therefore, the effects on the NF- $\mathrm{KB}$ activation and inflammatory cytokines induction might be resulted from lipopolysaccharides of S. typhimurium. On the other hand, our current study showed that $L$. acidophilus significantly induced TGF- $\beta$ /SMAD activity in intestinal cells. In addition to TGF- $\beta$ /MIR21 regulation on SMAD7 expression, $L$. acidophilus inactivates Jak1/Stat1 signaling to decrease SMAD7 expression, suggesting that Jak1/Stat1 may be involved in the regulation of $L$. acidophilus on $S$. typhimurium-induced SMAD7 expression [9]. It is necessary to further elucidate the detailed mechanisms of TGF- $\beta /$ SMAD regulation in human intestinal cells treated with $L$. acidophilus.

Microbes play a pivotal role in intestinal homeostasis, such as the uptake of nutrients and the generation of essential elements. However, pathogen infection may disrupt the homeostasis of intestinal microbes and cause acute gastroenteritis or chronic inflammatory diseases. Numerous clinical reports show that probiotics play beneficial roles on reducing incidence of gastrointestinal infectious diseases, including acute diarrhea, necrotizing enterocolitis and death [35-37]. Our study showed that TGF- $\beta 1 /$ MIR 21 signaling pathway may be involved in the suppressive effects of $L$. acidophilus on inflammation in intestinal cells during S. typhimurium infection. Previous reports also indicate that prebiotics activate immune system in animals during enteric pathogen infection [31]. However, our current study show that prebiotic (inulin) has no effect on diminishing inflammation in intestinal cells during S. typhimurium infection, suggesting inulin may preferentially modulate immune cells to reduce inflammation in vivo. Although the cell culture model may not be able to precisely reflect actual infection due to complicated nature of microbiota and host defense system in vivo, our study provide a potential mechanism of $L$. acidophilus on inflammation suppression in intestinal cells during S. typhimurium infection.

\section{Conclusion}

Our current study indicates that $L$. acidophilus induces TGF- $\beta$ /MIR21 expression to reduce NF-kB-activated inflammation caused by $S$. typhimurium in human intestinal Caco- 2 cells. The TGF- $\beta$ /MIR21 expression might be used as a marker to evaluate anti-inflammatory effects of other strains of Lactobacillus, which requires further study to determine the potential application.

\section{Additional files}

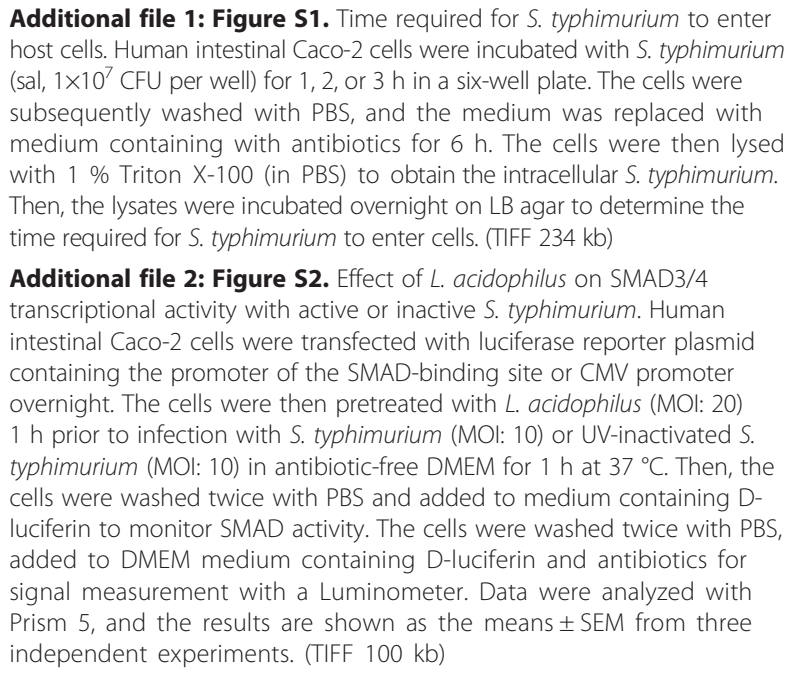

Additional file 2: Figure S2. Effect of L. acidophilus on SMAD3/4 transcriptional activity with active or inactive S. typhimurium. Human intestinal Caco-2 cells were transfected with luciferase reporter plasmid containing the promoter of the SMAD-binding site or CMV promoter overnight. The cells were then pretreated with L. acidophilus (MOI: 20) $1 \mathrm{~h}$ prior to infection with S. typhimurium (MOI: 10) or UV-inactivated S. typhimurium (MOl: 10) in antibiotic-free DMEM for $1 \mathrm{~h}$ at $37^{\circ} \mathrm{C}$. Then, the cells were washed twice with PBS and added to medium containing Dluciferin to monitor SMAD activity. The cells were washed twice with PBS, added to DMEM medium containing D-luciferin and antibiotics for signal measurement with a Luminometer. Data were analyzed with Prism 5, and the results are shown as the means \pm SEM from three independent experiments. (TIFF $100 \mathrm{~kb}$ )

\section{Abbreviations}

S. typhimurium: Salmonella typhimurium; L. acidophilus: Lactobacillus acidophilus; TGF- $\beta$ : Transforming growth factor beta; NF-kB: Nuclear Factor-kappa B; CFU: Colony-forming unit; IL-8: Chemokine (C-X-C motif) 
ligand 8; TNF-a: Tumor necrosis factor alpha; SMAD7: SMAD family member 7; MIR21: MicroRNA 21.

\section{Competing interests}

The authors declare that they have no competing interests

\section{Authors' contributions}

Conceived and designed the experiments: CWS and HWC. Performed the experiments: IFH, ICL, PFL, MFC and YCL. Analyzed the data: MFC, YDH and JJC. Contributed reagents/materials/analysis tools: IFH, ICL, HWC. Wrote the paper: CWS and IFH. All authors contributed to the final manuscript. All authors read and approved the final manuscript.

\section{Author details}

IFH and MFC: Attending physician at Kaohsiung Veterans General Hospital, Kaohsiung. YDH: assistant administrator at Kaohsiung Veterans General Hospital, Pingtung. ICL: nutritionist at Zuoying Branch of Kaohsiung Armed Forces General Hospital. YCL: research assistant at Kaohsiung Veterans General Hospital, Kaohsiung. PFL: Post-doctor at Kaohsiung Veterans General Hospital, Kaohsiung. JJC: professor at Tajen University. CLC: assistant professor at National Sun Yat-sen University. HWC: professor at Kaohsiung Medical University, Kaohsiung. CWS: associate research fellow at Kaohsiung Veterans General Hospital, Kaohsiung.

\section{Acknowledgments}

This work was supported by Kaohsiung Veterans General Hospital (VGHKS102-97 VGHNSU103-004 to I-Fei Huang, VGHKS100-125 to Chih-Wen Shu), Zuoying Branch of Kaohsiung Armed Forces General Hospital (ZBH 104-26 to I-Chun Lin), and Kaohsiung Medical University "Aim for the Top Universities Grant (KMU-TP103A33 to Hsueh-Wei Chang).

\section{Author details}

'Department of Pediatrics, Kaohsiung Veterans General Hospital, Kaohsiung, Taiwan. ${ }^{2}$ Faculty of Medicine, National Yang-Ming University, Taipei, Taiwan. ${ }^{3}$ Chung Hwa University of Medical Technology, Tainan, Taiwan. ${ }^{4}$ Diet and Nutrition Section, Zuoying Branch of Kaohsiung Armed Forces General Hospital, Kaohsiung, Taiwan. ${ }^{5}$ Department of Medical Education and Research, Kaohsiung Veterans General Hospital, Kaohsiung, Taiwan. ${ }^{6}$ Department of Dentistry, Kaohsiung Veterans General Hospital, Pingtung, Taiwan. ${ }^{7}$ Department of Pharmacy, Tajen University, Pingtung, Taiwan. ${ }^{8}$ Department of Biological Science, National Sun Yat-sen University, Kaohsiung, Taiwan. ${ }^{9}$ Doctoral Degree Program in Marine Biotechnology, National Sun Yat-sen University and Academia Sinica, Kaohsiung, Taiwan. ${ }^{10}$ Department of Biomedical Science and Environmental Biology, Kaohsiung Medical University, Kaohsiung, Taiwan. ${ }^{11}$ Research Center of Environmental Medicine, Kaohsiung Medical University, Kaohsiung, Taiwan.

\section{Received: 6 April 2015 Accepted: 2 October 2015}

\section{Published online: 07 October 2015}

\section{References}

1. Backhed F, Ding H, Wang T, Hooper LV, Koh GY, Nagy A, et al. The gut microbiota as an environmental factor that regulates fat storage. Proc Natl Acad Sci U S A. 2004;101(44):15718-23.

2. Turnbaugh PJ, Ley RE, Mahowald MA, Magrini V, Mardis ER, Gordon II. An obesity-associated gut microbiome with increased capacity for energy harvest. Nature. 2006;444(7122):1027-31.

3. Mundy R, MacDonald T, Dougan G, Frankel G, Wiles S. Citrobacter rodentium of mice and man. Cell Microbiol. 2005;7(12):1697-706.

4. Schiller LR. Infectious disease: A germy world-food-borne infections in 2009. Nat Rev Gastroenterol Hepatol. 2009;6(4):197-8.

5. Abrahams GL, Muller P, Hensel M. Functional dissection of SseF, a type III effector protein involved in positioning the salmonella-containing vacuole. Traffic. 2006;7(8):950-65.

6. Gewirtz AT, Rao AS, Simon Jr PO, Merlin D, Carnes D, Madara JL, et al. Salmonella typhimurium induces epithelial IL-8 expression via $\mathrm{Ca}(2+)$-mediated activation of the NF-kappaB pathway. J Clin Invest. 2000;105(1):79-92.

7. Kim MS, Yoon YS, Seo JG, Lee HG, Chung MJ, Yum DY. A study on the prevention of salmonella infection by using the aggregation characteristics of lactic Acid bacteria. Toxicol Res. 2013;29(2):129-35.
8. Brown SP, Le Chat L, Taddei F. Evolution of virulence: triggering host inflammation allows invading pathogens to exclude competitors. Ecol Lett. 2008;11(1):44-51.

9. Yang YJ, Chuang CC, Yang HB, Lu CC, Sheu BS. Lactobacillus acidophilus ameliorates $\mathrm{H}$. pylori-induced gastric inflammation by inactivating the Smad7 and NFkappaB pathways. BMC Microbiol. 2012;12:38.

10. McCabe LR, Irwin R, Schaefer L, Britton RA. Probiotic use decreases intestinal inflammation and increases bone density in healthy male but not female mice. J Cell Physiol. 2013;228(8):1793-8.

11. Dimitrijevic R, Ivanovic N, Mathiesen G, Petrusic V, Zivkovic I, Djordjevic $B$, et al. Effects of Lactobacillus rhamnosus LA68 on the immune system of C57BL/6 mice upon oral administration. J Dairy Res. 2014;81(2):202-7.

12. Ivanovic N, Minic R, Dimitrijevic L, Radojevic Skodric S, Zivkovic I, Djordjevic B. Lactobacillus rhamnosus LA68 and Lactobacillus plantarum WCFS1 differently influence metabolic and immunological parameters in high fat diet-induced hypercholesterolemia and hepatic steatosis. Food Funct. 2015;6(2):558-65.

13. Li C, Nie SP, Zhu KX, Ding Q, Li C, Xiong T, et al. Lactobacillus plantarum NCU116 improves liver function, oxidative stress and lipid metabolism in rats with high fat diet induced non-alcoholic fatty liver disease. Food Funct. 2014;5(12):3216-23.

14. Chiu YH, Lu YC, Ou CC, Lin SL, Tsai CC, Huang CT, et al. Lactobacillus plantarum MYL26 induces endotoxin tolerance phenotype in Caco-2 cells. BMC Microbiol. 2013;13:190.

15. Chiu YH, Lin SL, Tsai JJ, Lin MY. Probiotic actions on diseases: implications for therapeutic treatments. Food Funct. 2014;5(4):625-34.

16. Monteleone G, Pallone F, MacDonald TT. Smad7 in TGF-beta-mediated negative regulation of gut inflammation. Trends Immunol. 2004;25(10):513-7.

17. Bitzer M, von Gersdorff G, Liang D, Dominguez-Rosales A, Beg AA, Rojkind M, et al. A mechanism of suppression of TGF-beta/SMAD signaling by NF-kappa B/RelA. Genes Dev. 2000;14(2):187-97.

18. Foye OT, Huang IF, Chiou CC, Walker WA, Shi HN. Early administration of probiotic Lactobacillus acidophilus and/or prebiotic inulin attenuates pathogen-mediated intestinal inflammation and Smad 7 cell signaling. FEMS Immunol Med Microbiol. 2012;65(3):467-80.

19. Li Q, Zhang D, Wang Y, Sun P, Hou X, Larner J, et al. MiR-21/Smad 7 signaling determines TGF-beta1-induced CAF formation. Sci Rep. 2013;3:2038.

20. Wang T, Zhang L, Shi C, Sun H, Wang J, Li R, et al. TGF-beta-induced miR-21 negatively regulates the antiproliferative activity but has no effect on EMT of TGF-beta in HaCaT cells. Int J Biochem Cell Biol. 2012;44(2):366-76.

21. Kim YJ, Hwang SJ, Bae YC, Jung JS. MiR-21 regulates adipogenic differentiation through the modulation of TGF-beta signaling in mesenchymal stem cells derived from human adipose tissue. Stem cells (Dayton, Ohio). 2009;27(12):3093-102.

22. Dong J, Jimi E, Zeiss C, Hayden MS, Ghosh S. Constitutively active NF-kappaB triggers systemic TNFalpha-dependent inflammation and localized TNFalphaindependent inflammatory disease. Genes Dev. 2010;24(16):1709-17.

23. Hoffmann E, Dittrich-Breiholz O, Holtmann H, Kracht M. Multiple control of interleukin-8 gene expression. J Leukoc Biol. 2002;72(5):847-55.

24. Karimi R, Azizi MH, Ghasemlou M, Vaziri M. Application of inulin in cheese as prebiotic, fat replacer and texturizer: A review. Carbohydr Polym. 2015;119C:85-100.

25. Godinez I, Raffatellu M, Chu H, Paixao TA, Haneda T, Santos RL, et al. Interleukin-23 orchestrates mucosal responses to Salmonella enterica serotype Typhimurium in the intestine. Infect Immun. 2009;77(1):387-98.

26. Malago JJ, Nemeth E, Koninkx JF, Tooten PC, Fajdiga S, van Dijk JE. Microbial products from probiotic bacteria inhibit Salmonella enteritidis 857-induced IL-8 synthesis in Caco-2 cells. Folia Microbiol (Praha). 2010;55(4):401-8.

27. Bested AC, Logan AC, Selhub EM. Intestinal microbiota, probiotics and mental health: from Metchnikoff to modern advances: Part I - autointoxication revisited. Gut Pathog. 2013;5(1):5.

28. Monteleone G, Fantini MC, Onali S, Zorzi F, Sancesario G, Bernardini S, et al. Phase I clinical trial of Smad7 knockdown using antisense oligonucleotide in patients with active Crohn's disease. Mol Ther. 2012;20(4):870-6.

29. Roberfroid M. Prebiotics: the concept revisited. J Nutr. 2007;137 (3 Suppl 2):830S-7S.

30. Scholz-Ahrens KE, Ade P, Marten B, Weber P, Timm W, Acil Y, et al. Prebiotics, probiotics, and synbiotics affect mineral absorption, bone mineral content, and bone structure. J Nutr. 2007;137(3 Suppl 2):838S-46S. 
31. Lomax AR, Calder PC. Prebiotics, immune function, infection and inflammation: a review of the evidence. Br J Nutr. 2009;101(5):633-58.

32. Geier MS, Butler RN, Howarth GS. Inflammatory bowel disease: current insights into pathogenesis and new therapeutic options; probiotics, prebiotics and synbiotics. Int J Food Microbiol. 2007;115(1):1-11.

33. Hedin C, Whelan K, Lindsay JO. Evidence for the use of probiotics and prebiotics in inflammatory bowel disease: a review of clinical trials. Proc Nutr Soc. 2007:66(3):307-15.

34. Zenhom M, Hyder A, de Vrese M, Heller KJ, Roeder T, Schrezenmeir J. Prebiotic oligosaccharides reduce proinflammatory cytokines in intestinal Caco-2 cells via activation of PPARgamma and peptidoglycan recognition protein 3. J Nutr. 2011;141(5):971-7.

35. Sazawal S, Hiremath G, Dhingra U, Malik P, Deb S, Black RE. Efficacy of probiotics in prevention of acute diarrhoea: a meta-analysis of masked, randomised, placebo-controlled trials. Lancet Infect Dis. 2006;6(6):374-82.

36. Lin JS, Chiu YH, Lin NT, Chu CH, Huang KC, Liao KW, et al. Different effects of probiotic species/strains on infections in preschool children: A doubleblind, randomized, controlled study. Vaccine. 2009;27(7):1073-9.

37. Lin HC, Hsu CH, Chen HL, Chung MY, Hsu JF, Lien Rl, et al. Oral probiotics prevent necrotizing enterocolitis in very low birth weight preterm infants: a multicenter, randomized, controlled trial. Pediatrics. 2008;122(4):693-700.

\section{Submit your next manuscript to BioMed Central and take full advantage of:}

- Convenient online submission

- Thorough peer review

- No space constraints or color figure charges

- Immediate publication on acceptance

- Inclusion in PubMed, CAS, Scopus and Google Scholar

- Research which is freely available for redistribution 